\title{
Enrichment of Mutants of Mucor racemosus by Differential Freeze-killing
}

\author{
By JULIUS PETERS AND PAUL S. SYPHERD \\ Department of Medical Microbiology, California College of Medicine, \\ University of California, Irvine, California 92717, U.S.A.
}

(Received 31 August 1977)

\begin{abstract}
An efficient technique for the enrichment of mutants of Mucor racemosus by differential freeze-killing is described. Ungerminated spores were resistant to freeze-killing. During germination susceptibility increased up to a maximum coinciding with the appearance of germ tubes. Under optimum conditions a thousandfold preferential survival of growthlimited spores over actively germinating spores was obtained. Using this differential killing, cultures treated with mutagen were enriched with respect to auxotrophic and temperaturesensitive mutants, with the desired mutants constituting a large fraction of the survivors of the freeze-thaw treatments. This technique may be applicable to mutant enrichment in other filamentous fungi.
\end{abstract}

\section{INTRODUCTION}

Vegetative growth of Mucor racemosus occurs in two alternative forms: aerobically $M$. racemosus grows as a filamentous fungus but in $\mathrm{CO}_{2}$ it grows as a budding yeast (Bartnicki-Garcia, 1963). It was desirable to isolate both auxotrophic and conditional mutants for the investigation of this dimorphism.

We describe here a simple and effective technique for selecting auxotrophic and temperature-sensitive mutants of $M$. racemosus based on our observation that survival after freezing and thawing is influenced by the developmental stage at which the treatment is carried out. Although no published data are available, it has been reported that metabolically active conidia of Neurospora crassa are much more sensitive to freezing damage than are the inactive conidia (Leef \& Mazur, 1975). A cryobiological method exploiting this difference for the selection of mutants of $N$. crassa has been previously outlined (Leef \& Gaertner, 1975).

\section{METHODS}

Fungal strains. Mucor racemosus (Fresenius) syn. Mucor lusitanicus (Brunderkin) ATCC 1216B was used as the parental strain. The leucine auxotroph leu-2A and the adenine auxotroph ade-1B used in the reconstruction experiments were induced and selected by the procedure described in this paper.

Media. Yeast extract/peptone/glucose (YPG) broth (Bartnicki-Garcia \& Nickerson, 1962) was used as the complex medium. The minimal medium contained Difco yeast nitrogen base [without amino acids or $\left.\left(\mathrm{NH}_{4}\right)_{2} \mathrm{SO}_{4}\right]\left(0.5 \mathrm{~g}^{-1}\right)$, glucose $\left(20 \mathrm{~g} \mathrm{l}^{-1}\right)$ and glutamate, aspartate, alanine and $\mathrm{NH}_{4} \mathrm{Cl}$ (each at a final concentration of $10 \mathrm{~mm}$ ). For growth of auxotrophs, the minimal medium was supplemented with leucine or adenine at a final concentration of $1.0 \mathrm{~mm}$. Liquid media were adjusted to $\mathrm{pH} 4.5$ with $1.5 \mathrm{M}-\mathrm{H}_{2} \mathrm{SO}_{4}$. Plating media solidified with $15 \mathrm{~g}$ agar $\mathbf{1}^{-1}$ were adjusted to $\mathrm{pH} 5.8$ to avoid acid hydrolysis of the agar during autoclaving.

Culture conditions. Spores were obtained from mycelia grown aerobically for 4 to $7 \mathrm{~d}$ on YPG or minimal plating medium. For aerobic germination, $20 \mathrm{ml}$ liquid medium was inoculated with washed spores at $5 \times 10^{5} \mathrm{ml}^{-1}$ and incubated with vigorous shaking at 20 to $22{ }^{\circ} \mathrm{C}$. For the isolation of temperature-sensitive mutants, $37^{\circ} \mathrm{C}$ was used as the restrictive temperature. For anaerobic growth, liquid cultures were flushed 
continually with $100 \% \mathrm{CO}_{2}$ at a flow rate of $0.5 \mathrm{vol}$. gas $\mathrm{min}^{-1}$ per vol. culture. Viability (colony-forming ability) was determined under anaerobic conditions in BBL GasPak anaerobic jars using disposable $\mathrm{CO}_{2} / \mathrm{H}_{2}$ generating envelopes (Brewer \& Allgeier, 1966). Cultures were diluted in $5 \%(\mathrm{v} / \mathrm{v})$ glycerol for plating. Colony counts were made on YPG or minimal medium after 4 or $7 \mathrm{~d}$ incubation, respectively.

Mutagenesis. An adaptation of the procedure developed for the mutagenesis of spores of Streptomyces coelicolor was used (Delic, Hopwood \& Friend, 1970). Freshly harvested spores were washed and resuspended at $2 \times 10^{8} \mathrm{ml}^{-1}$ in sterile distilled water. Four mg $N$-methyl- $N^{\prime}$-nitro- $N$-nitrosoguanidine (NTG) was dissolved in $17 \mathrm{ml}$ sterile distilled water at $30^{\circ} \mathrm{C}$. Spore suspension $(1 \mathrm{ml})$ was added to the NTG solution, followed immediately by $2.0 \mathrm{ml} 0.50 \mathrm{M}$-Tris/maleic acid buffer, $\mathrm{pH} 9$. The suspension, containing a final concentration

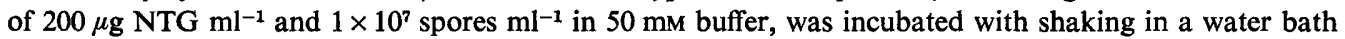
at $30^{\circ} \mathrm{C}$. After incubation for $30 \mathrm{~min}$ the spores were removed from the mutagen by filtration, washed and then resuspended in YPG broth at $2 \times 10^{6} \mathrm{ml}^{-1}$. The viability of the mutagen-treated spores was reduced to about $1 \%$ of that of untreated controls. Thus, suspensions of treated spores in broth contained $2 \times 10^{4}$ viable spores $\mathrm{ml}^{-1}$. Several such cultures were prepared in each experiment in order to obtain mutants of independent origin.

Mutant enrichment. NTG-treated spores were germinated and grown in the yeast phase under $\mathrm{CO}_{2}$. After incubation for $5 \mathrm{~d}$ in YPG broth the cultures contained $1 \times 10^{6}$ to $3 \times 10^{6}$ cells $\mathrm{ml}^{-1}$. The cells were harvested and suitable plating media were seeded with $5 \times 10^{5}$ washed cells per plate. Plating conditions were used which permitted the growth and sporulation of both wild-type and mutant cells, i.e. appropriately supplemented minimal medium and YPG medium at the permissive temperature for the growth of auxotrophs and temperature-sensitive mutants, respectively. The spores harvested from these plates after aerobic growth for 4 to $7 \mathrm{~d}$ were used to inoculate $20 \mathrm{ml}$ medium at $5 \times 10^{5}$ spores $\mathrm{ml}^{-1}$. The medium and incubation conditions allowed aerobic germination of wild-type spores but prevented the germination of mutant spores. Thus, unsupplemented minimal medium was used to select auxotrophs, and YPG broth with incubation at $37^{\circ} \mathrm{C}$ was used to select temperature-sensitive mutants. Cultures were harvested when over $90 \%$ of the spores had germinated and had entered early hyphal development. For YPG and minimal medium this stage was reached after 11 and $15 \mathrm{~h}$, respectively. The cells were washed and resuspended at $2 \times 10^{7} \mathrm{ml}^{-1}$ in sterile distilled water. Portions $(2 \mathrm{ml})$ of the cell suspension were frozen in $8.5 \mathrm{ml}$ test tubes at $-20^{\circ} \mathrm{C}$ in a standard laboratory freezer. After $12 \mathrm{~h}$ the suspension was thawed in a water bath at $20^{\circ} \mathrm{C}$ and was then subjected to a second freezing and thawing treatment. A portion of the suspension after this cycle of two freeze-thaw treatments was plated at the appropriate dilutions and incubated anaerobically to determine viability and the frequency of mutants among the survivors. Plates with $3 \times 10^{1}$ to $1 \times 10^{2}$ yeast-phase colonies were replica-plated on to supplemented and unsupplemented minimal medium. Colonies of presumptive mutants were picked and tested by restreaking on appropriate media. Another portion of the cell suspension was subjected to a second cycle of freeze-thaw treatments. Portions $(0.2 \mathrm{ml})$ of the undiluted suspension from the first cycle were plated and incubated aerobically to produce spores. These spores were in turn germinated in unsupplemented minimal medium, frozen and thawed twice, plated and incubated anaerobically on supplemented minimal medium. Viability and the frequency of mutants were determined as before. Temperature-sensitive mutants were detected by shifting yeast-phase colonies grown anaerobically at 20 to $22{ }^{\circ} \mathrm{C}$ to aerobic conditions at $37{ }^{\circ} \mathrm{C}$; mutants whose growth was blocked by the restrictive temperature remained in the yeast-phase while the wild-type colonies grew and changed to the mycelial morphology.

\section{RESULTS AND DISCUSSION}

The survival of $M$. racemosus after freezing and thawing varied with the growth stage (Fig. 1). Initially, about $40 \%$ of the ungerminated spores remained viable after freezing. During germination the susceptibility of the spores increased sharply. The most sensitive stage, coinciding with the appearance of germ tubes, was reached after 9 to $12 \mathrm{~h}$; at this stage, survival was reduced to 0.5 to $0.7 \%$ of the untreated control. As hyphal development continued the more mature hyphae became less sensitive to freezing.

On the basis of this enhanced sensitivity during germination, auxotrophic derivatives should survive preferentially when germination is blocked by the omission of a required growth supplement. A reconstruction experiment was performed comparing the survival following freezing and thawing of a leucine auxotroph (leu-2A) after incubation for $12 \mathrm{~h}$ in unsupplemented minimal medium or in minimal medium supplemented with leucine (Fig. 2). When harvested, the unsupplemented culture contained predominantly ungerminated spores and the supplemented culture contained predominantly germlings. After five successive freezing and thawing treatments about $10 \%$ of the spores from the unsupple- 


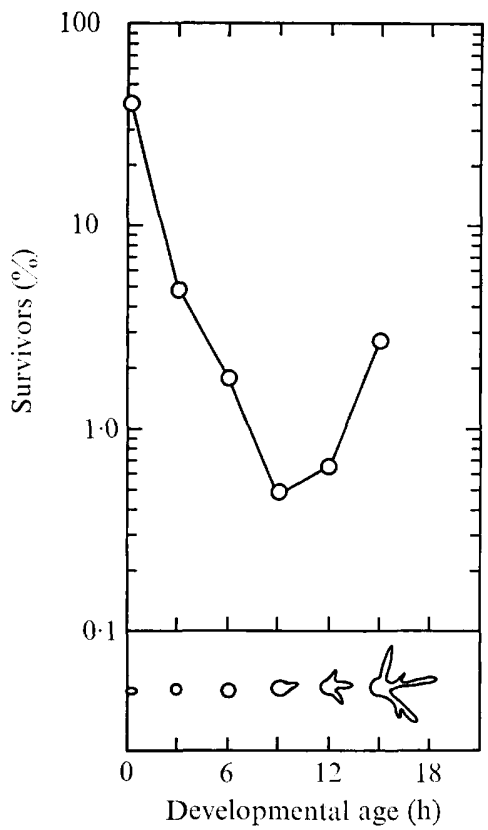

Fig. 1

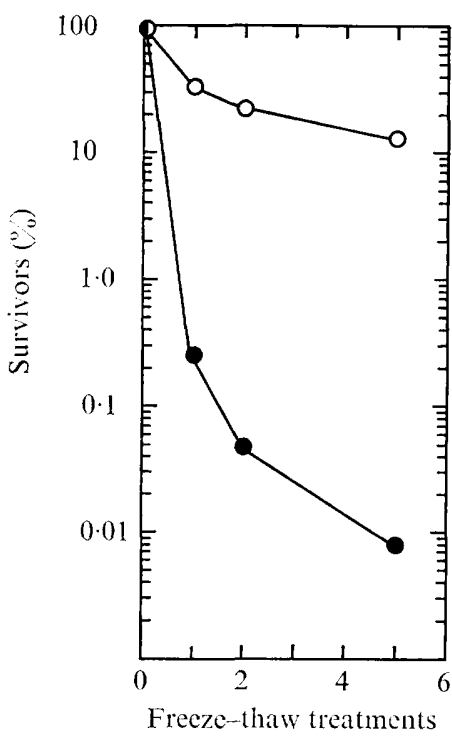

Fig. 2

Fig. 1. Survival after freezing as a function of developmental stage. Spores of the wild-type strain ATCC 1216B were germinated aerobically in minimal medium. Samples $(0.5 \mathrm{ml})$ were taken from the culture at $3 \mathrm{~h}$ intervals, diluted 10-fold into sterile distilled water and frozen for $12 \mathrm{~h}$. Colony-forming ability before and after freezing was determined on YPG medium. The diagram at the foot of the figure relates developmental stage to incubation time. For each point in Figs 1 to 3 plating was done in triplicate and each experiment was performed with duplicate cultures.

Fig. 2. Differential survival in actively growing and growth-limited cultures. Spores of the leucine auxotroph leu-2A were incubated in minimal medium supplemented with $1 \mathrm{~mm}$-leucine $(\odot)$ and in unsupplemented minimal medium $(O)$. After aerobic incubation for $12 \mathrm{~h}$, cultures were harvested by filtration and resuspended in distilled water. Colony-forming ability was determined on YPG medium before and after one, two, and five successive freezing and thawing treatments.

mented culture remained viable compared with less than $0.01 \%$ of the germlings from the culture supplemented with leucine. The most efficient differential killing occurred during the first two freezing and thawing treatments.

The enrichment of auxotrophic mutants through their preferential survival was directly demonstrated using a mixed culture of an adenine-requiring mutant (ade-1B) and the wildtype strain. This auxotroph produces pink pigmented colonies on media containing low concentrations of adenine as found in some adenine auxotrophs of other fungi (Fisher, 1969). Thus, colonies of the mutant can be distinguished from those of the wild type by their pigmentation. $\mathrm{Ade}^{-}$and $\mathrm{Ade}^{+}$spores were mixed to give an initial frequency of about $3 \% \mathrm{Ade}^{-}$mutants and were then used to inoculate minimal medium supplemented with adenine. After five successive freezing and thawing treatments the viability of the cultures was reduced to $0.01 \%$ of the untreated control but the ratio of $\mathrm{Ade}^{-}$to $\mathrm{Ade}^{+}$cells remained essentially unchanged, indicating that the mutant and the wild-type strain were killed at the same rate (Fig. $3 a$ ). However, when the experiment was repeated in minimal medium without adenine the wild-type cells were preferentially killed. The frequency of the mutant increased from $0.3 \%$ in the untreated culture to $65 \%$ of the survivors after five freezing and thawing treatments (Fig. $3 b$ ).

The enrichment technique developed in the foregoing experiments was applied to the de novo isolation of other mutants. Selection was carried out for adenine and leucine auxo- 


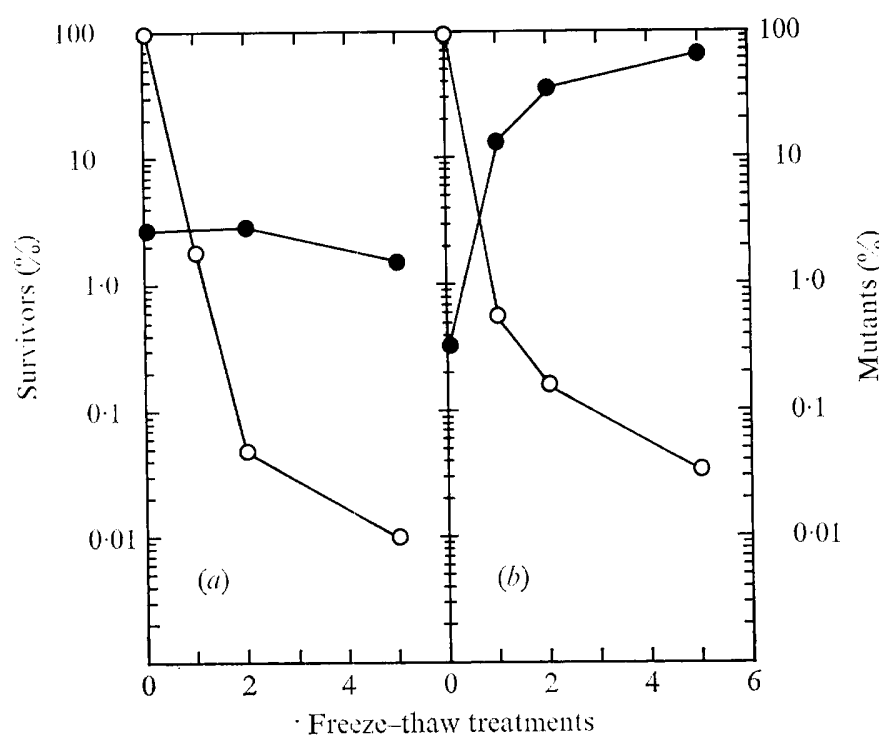

Fig. 3. Survival of an auxotrophic mutant in mixed culture with the wild-type strain. Spores of the adenine auxotroph ade-1B were incubated in mixed cultures with spores of the wild-type strain in minimal medium supplemented with 1 mm-adenine $(a)$ and in unsupplemented minimal medium (b). After aerobic incubation for $12 \mathrm{~h}$, cultures were harvested by filtration, resuspended in distilled water and subjected to freezing and thawing treatments. Colony-forming ability $(O)$ and the frequency of mutants among the survivors $(0)$ were determined on YPG medium.

\section{Table 1. Enrichment of auxotrophic and temperature-sensitive mutants by freezing and thawing}

NTG-treated spores were outgrown in YPG broth under $\mathrm{CO}_{2}$ for $5 \mathrm{~d}$ in the yeast phase. Six plates each seeded with $5 \times 10^{5}$ cells were incubated aerobically under permissive conditions. After aerobic growth for 4 to $7 \mathrm{~d}$ the spores were harvested and germinated under restrictive conditions. The germinated cultures were frozen and thawed twice in succession. A portion of the survivors of this treatment was used to seed plates for a second cycle of selection in which the above treatment was repeated. The frequencies of mutant and viable cells were determined at each step of the enrichment.

\section{Phenotype of mutants}

Adenine-requiring

Leucine-requiring

Temperature-sensitive

\begin{tabular}{ccc} 
Mutants & Birst cycle of selection \\
\cline { 2 - 2 }$(\%)$ & $\begin{array}{c}\text { Survivors } \\
(\%)\end{array}$ & $\begin{array}{c}\text { Mutants } \\
(\%)\end{array}$ \\
$0.0082 *$ & 0.060 & $2.8 \dagger$ \\
- & 0.11 & 1.8 \\
3.3 & 0.53 & 26
\end{tabular}

Second cycle of selection

$\begin{array}{cc}\begin{array}{c}\text { Survivors } \\ (\%)\end{array} & \begin{array}{c}\text { Mutants } \\ (\%)\end{array} \\ 0.33 & 17 \dagger \\ 1.7 & 75 \\ - & -\end{array}$

* Only the pigmented class of adenine auxotrophs was scored, by inspection of $5 \times 10^{4}$ colonies.

$\uparrow$ Both pigmented and non-pigmented adenine auxotrophs were scored, by replica-plating of $5 \times 10^{2}$ colonies. About one-third of the adenine auxotrophs were pigmented.

trophs and temperature-sensitive mutants. Other auxotrophs were eliminated by plating the cells obtained after mutagenesis on to minimal medium supplemented only with adenine or leucine. The frequency of auxotrophs was 2 to $3 \%$ after the first cycle of selection and after the second cycle the desired mutants constituted a large fraction of the survivors (Table 1). About a quarter of the survivors were temperature-sensitive mutants after the first cycle of selection although in this case the initial frequency of the mutants (about $3 \%$ ) was also high (Table 1).

Selective enrichment of mutants by freezing and thawing may be effective for a whole range of filamentous fungi. A cryobiological method for the enrichment of mutant conidia 
in $N$. crassa has been outlined and its use proposed either alone or in combination with enrichment by filtration or by inositol-less death (Leef \& Gaertner, 1975). Preliminary experiments indicate that a method similar to that developed for Mucor is also effective for the selection of mutants of Phycomyces blakesleeanus (D. Radin, personal communication), whereas other enrichment techniques have not been successful in this organism (CerdaOlmedo, 1974).

This work was supported in part by a grant (GM 18293) from the National Institute of General Medical Sciences, National Institutes of Health, U.S.A.

\section{REFERENCES}

BartNiCKI-Garcia, S. (1963). Symposium on biochemical bases of morphogenesis in fungi. III. Mold-yeast dimorphism of Mucor. Bacteriological Reviews 27, 293-304.

Bartnicki-Garcia, S. \& Nickerson, W. J. (1962). Induction of yeastlike development in Mucor by carbon dioxide. Journal of Bacteriology 84, 829840.

Brewer, J. H. \& Allgeier, D. L. (1966). Safe selfcontained carbon dioxide-hydrogen anaerobic system. Applied Microbiology 14, 985-988.

Cerda-Olmedo, E. (1974). Phycomyces. In Handbook of Genetics, vol. 1, p. 347. Edited by R. C. King. New York: Plenum Press.

Delic, V., Hopwood, D. A. \& Friend, E. J. (1970).
Mutagenesis by $N$-methyl- $N^{\prime}$-nitro- $N$-nitrosoguanidine (NTG) in Streptomyces coelicolor. Mutation Research 9, 167-182.

FisHER, C. R. (1969). Enzymology of the pigmented adenine-requiring mutants of Saccharomyces and Schizosaccharomyces. Biochemical and Biophysical Research Communications 34, 306-310.

LeEF, J. L. \& GAeRTNeR, F. H. (1975). A cryobiological method for isolating fungal mutants. Cryobiology 12, 584 (Abstract).

Leef, J. L. \& MAZuR, P. (1975). The physiological response of conidia of Neurospora crassa to low temperature in dehydrated or germinated state. Cryobiology 12, 568 (Abstract). 